\title{
Diagnosing Autonomic Nervous System Disorders- Existing Guidelines and Future Perspectives
}

\author{
Heinz Lahrmann, MD, ${ }^{1}$ Isabel Rocha, MD, ${ }^{2}$ Walter Struhal, MD, ${ }^{3}$ Roland D Thijs, MD, $\mathrm{PhD}^{4}$ and $\mathrm{Max} \mathrm{Hilz,} \mathrm{MD,} \mathrm{PhD}^{5}$

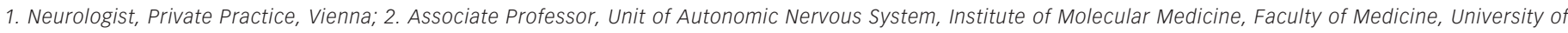 \\ Lisbon; 3. Neurologist, Autonomic Unit, Department of Neurology and Psychiatry, General Hospital of the City of Linz; 4. Neurologist, Department of Neurology and

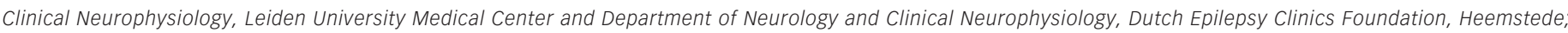 \\ 5. Professor of Neurology, Medicine and Psychiatry, Department of Neurology, University of Erlangen-Nuremberg and
}

Departments of Neurology, Medicine and Psychiatry, New York University

\begin{abstract}
Primary and secondary autonomic nervous system (ANS) disorders often have a severe adverse effect on the quality of life of patients. Diagnostics for ANS disorders are under represented, despite their common occurrence. Precise history taking is of key importance for ANS evaluation: it may help to rule out differential diagnoses and provide important clues to the underlying ANS disorder. In fact, in conjunction with additional bedside tests, it can achieve a clear diagnosis. The analysis of heart rate variability and the results of the standardized tests that make up the Ewing battery are important means of evaluating the parasympathetic and sympathetic nervous system. In addition, sudomotor testing can be used to evaluate cholinergic sympathetic function, and the spontaneous baroreceptor reflex can be assessed using new computerized techniques. These tests provide valuable information on cardiovascular autonomic control. This paper presents a structured review of current standard techniques for diagnosing ANS disorders.
\end{abstract}

\section{Keywords}

Autonomic testing, sudomotor function, baroreflex sensitivity, heart rate variability, Ewing battery

Disclosure: The authors have no conflicts of interest to declare.

Received: November 12, 2010 Accepted: January 17, 2011 Citation: US Neurology, 2011;7(1):55-9 DOI: 10.17925/USN.2011.07.01.55

Correspondence: Heinz Lahrmann, MD, Hegergasse 21/16, A-1030 Wien, Austria. E: office@lahrmann.at

Disorders of the autonomic nervous system (ANS) can be of central or peripheral origin, and may be primary illnesses (e.g. primary autonomic failure, multiple system atrophy) or develop secondary to other diseases (e.g. Parkinson's disease, diabetes, stroke, Guillain Barré syndrome). The anatomical and functional organization of the ANS is quite complex, involving structures within the brain, spinal cord and peripheral nervous system. Many feedback loops control homeodynamics and vital functions such as blood pressure (BP), heart rate (HR), ventilation, body temperature and blood gas allostasis.

To diagnose ANS disorders, it is of crucial importance to select and test the most compromized structures. In most diseases involving the ANS more than one diagnostic test is needed, and this should be selected after taking a symptom-guided patient history. A questionnaire-based survey revealed that cardiovascular and sudomotor tests are the diagnostic tests most frequently used in European autonomic laboratories. ${ }^{1}$ These are also integrated in many commercially-available electromyography (EMG) devices. Results must, however, be interpreted with great caution, particularly when comparing them with published normative data.

Many questions regarding cardiovascular regulation can be addressed using simple bedside tests such as the schellong test. If the results are inconclusive or more detailed information is needed a set of standardized tests (the Ewing battery) are available. The complexity of cardiovascular dynamics may be analyzed using sophisticated methods in time and frequency domains. The baroreflex represents one of the most important regulatory mechanisms for $\mathrm{BP}$ control and its function can be assessed by various techniques. Testing sudomotor function provides a good measure of cholinergic sympathetic integrity and may be used clinically for early diagnosis of small fiber neuropathy.

\section{History Taking}

Diligent history taking is the cornerstone of autonomic evaluation and can avoid the need for additional testing in many patients. The most frequently encountered clinical presentation in the autonomic field concerns transient loss of consciousness (TLOC). TLOC is defined as an apparent loss of consciousness with rapid onset, short duration and spontaneous and complete recovery. ${ }^{2,3}$ TLOC comprises a differential diagnostic group including syncope, generalized epileptic seizures, psychogenic TLOC as well as a number of rare causes. A thorough step-by-step history of as many attacks as possible needs to be taken from patients and eyewitnesses, allowing attending physicians to make a diagnosis based on initial evaluation (including history, physical examination and electrocardiogram [ECG]) in $63 \%$ of patients with TLOC, 
with an overall diagnostic accuracy of $88 \%{ }^{4}$ In most cases, a diagnosis of reflex syncope can thus be made without additional autonomic investigation; however, in case of diagnostic doubt, autonomic testing may be indicated.

Before ordering any kind of autonomic testing, a clear hypothesis should be formulated. For example, head-up tilt testing (HUT) is only helpful for diagnosing reflex syncope or orthostatic hypotension. Rarely, HUT may provoke psychogenic seizures, but other causes of TLOC—such as cardiac syncope and epileptic seizures-will not. Thus, in the evaluation of a patient with syncope while jogging, HUT is severely contraindicated, since the circumstances favor a cardiac cause, and ordering an autonomic test may result in a substantial delay in diagnosis. It also should be noted that additional investigations may confuse the diagnosis. For example, conducting HUT or the Ewing battery in elderly patients will very likely detect orthostatic hypotension $(\mathrm{OH})$, since $\mathrm{OH}$ affects up to $30 \%$ of all elderly people. ${ }^{3,5}$ However, the majority of cases are asymptomatic, therefore it is crucial to obtain more evidence before assuming causality. In patients with TLOC and $\mathrm{OH}$ it is necessary to establish whether clinical events were typically provoked by prolonged standing, cessation of exercise (post-exercise hypotension) and ingestion of a meal (post-prandial hypotension) ${ }^{2,3}$ Again, it is important to detail as many events as possible in order to recognize a clear pattern. Additionally, during the autonomic examination the patient should be questioned whether he or she experiences his or her typical symptoms.

\section{Bedside Testing}

After taking a precise history, there are simple means of investigating ANS without a sophisticated autonomic laboratory. TLOC caused by cardiac syncope has a high risk of mortality, therefore it is of utmost importance to first rule out this diagnosis. A simple 12-lead ECG can confirm the presence of bifascicular block, inadequate sinus bradycardia, pre-excited QRS complex, QT interval abnormities, negative T waves in right precordial leads, epsilon waves and ventricular late potentials. ${ }^{2}$ These patients should be referred to a cardiologist immediately.

Standard somatic and neurologic examinations are required, particularly when orthostatic hypotension is suspected. Dehydration might add to the development of orthostatic hypotension or reflex syncope. Polyneuropathy of the somatic nerves might accompany autonomic neuropathy. Parkinsonian signs might hint at the diagnosis of an alpha-synucleinopathy.

Finally, an inexpensive standing test can add valuable information. The schellong test is commonly used but, to save time, the protocol can be changed. The patient rests in the supine position for five to 10 minutes. $\mathrm{BP}$ and pulse rate measurements are taken regularly until a steady state is reached. These data are important as the starting point for further analysis. The patient moves to an upright position and another measurement is taken immediately and then at least every minute for up to 10 minutes. The patient is asked to report symptoms like dizziness, fatigue, headache and nausea. A decrease in BP of $20 \mathrm{mmHg}$ systolic and/or $10 \mathrm{mmHg}$ diastolic within three minutes of becoming upright gives a diagnosis of orthostatic hypotension. ${ }^{6}$ A pulse rate increase from supine to standing of more than 30 beats per minute, or to greater than 120 beats per minute, suggests postural tachycardia syndrome (PoTS). In only a few patients, the standing test provokes reflex syncope, and in these patients syncope typically occurs with prolonged standing. A study in 67 Austrian army recruits found the standing test to have a sensitivity of $61 \%$ and a specificity of $100 \%$ for PoTS. ' A sensitivity of $31 \%$ and a specificity of $100 \%$ for reflex syncope were noted in the same study.

Using these simple means, autonomic screening can be available on every ward, or in the outpatient department, allowing diagnoses to be made without the need to wait for additional tests.

\section{Cardiovascular Autonomic Testing}

\section{Autonomic Challenge Maneuvers (Ewing Battery)}

The cardiovascular and the respiratory systems play key roles in the maintenance of homeodynamics and there exist close similarities in the central organization of both. Reflex regulation of the cardiovascular system involves the activation of different types of receptors located mainly within the heart and blood vessels. Information collected is integrated in the brain stem area and, through negative feedback mechanisms, an appropriate response is generated and is conveyed to the target organs by the peripheral autonomic nervous system.

Autonomic testing has a clear focus on the cardiovascular system and its interactions with the respiratory system, due to the ease of non-invasive recording of cardiovascular variables. Based on this premise, a standard battery of autonomic challenge maneuvers was suggested by Ewing and Clarke. ${ }^{8}$ The Valsalva maneuver and active standing and deep breathing are the most valuable tests for clinical evaluations. Many other tests activate sympathetic or parasympathetic responses, including the cold pressor test, the cold face test, the sustained hand grip test, squatting, coughing and mental arithmetic. ${ }^{9}$ It should be stressed that no single test can provide a global assessment of autonomic function. The normative values of these tests depend upon a large number of factors, including: the specific laboratory conditions (e.g. room temperature, instrumentation); the protocol followed (e.g. duration of the stimulus, body position during and prior to testing); and patient-related factors (e.g. age, medication, consumption of caffeine, etc.) Care must therefore be taken when interpreting individual results on the basis of published normative values. From a clinical point of view it should be stressed that autonomic challenge maneuver tests are directed towards the determination of autonomic failure, as may be seen in the context of orthostatic intolerance. The Ewing battery is usually not helpful for detecting autonomic overactivity as seen in reflex syncope. For these patients, HUT may be indicated.

\section{Valsalva Maneuver}

This assesses the sympathetic and the parasympathetic reaction to baroreflex activation. The subject is asked to exhale into an almost occluded mouthpiece and to maintain an expiratory pressure of $40 \mathrm{mmHg}$ for 15 seconds. A very small hole in the mouthpiece keeps the glottis open. The test is divided into four phases. Phase 1 occurs during the first two to three seconds of the forced expiration and is associated with a brief decrease in HR and increase in BP due to mechanical compression of the aorta. In phase 2, BP first decreases and then increases in the latter part of the phase. Phase 3 describes the first one to two seconds after release of the expiratory strain with a consecutive decline in $\mathrm{BP}$ and increase in HR. Finally, phase 4 is associated with a BP overshoot due to 
persistent sympathetic activity together with normalization of venous return. The increased BP mediates baroreflex-induced bradycardia and is quantified using the Valsalva ratio (i.e. the ratio of the highest HR during expiration to the lowest HR during the first 20s after the release of strain). Results depend on the position, age and gender of the subject as well as the duration and intensity of the expiratory pressure. In patients with autonomic dysfunction, typically there is a loss of both the BP overshoot and the reflex bradycardia.

\section{Deep (Metronomic) Breathing}

Respiratory sinus arrhythmia with inspiratory acceleration and expiratory slowing of HR depends on the breathing rate and is maximal at a rate of five to six breaths/minute. Changes of HR with deep respiration can be considered a parameter of parasympathetic cardiac control. Usually, HR variability is assessed with the patient breathing metronomically at a rate of six cycles/minute for three minutes. The minimum and maximum HR of the averaged five largest consecutive responses are determined, and the difference between maximum and minimum HR and the expiratory/inspiratory ratio are calculated. HR variability during deep breathing is influenced by the position of the test subject, the rate and depth of breathing, hypocapnia, sympathetic activity, bodyweight and use of salicylates and other drugs. Decreased HR variability is an important indicator of cardiac vagal denervation but it should be noted that normative values decrease with age and with increasing resting HR.

\section{Active Standing}

To evaluate the initial cardiac responses to active standing up, Ewing introduced the 30/15 HR ratio. This is the ratio between the shortest $R$ wave to $R$ wave (RR) interval around the 15th heart beat and the longest RR interval around the 30th heart beat after standing up. Atropine abolishes the bradycardiac response, indicating that the 30/15 ratio is a parameter of cardiovagal function. In patients without secondary relative bradycardia, responses can be quantified by calculating the difference between the baseline HR and the highest HR occurring within 15 seconds of standing up. Normal values for both methods are age dependent. According to the Consensus Committee of the American Autonomic Society and the American Academy of Neurology, ${ }^{10}$ a fall in systolic pressure of at least $20 \mathrm{mmHg}$ or in diastolic pressure of at least $10 \mathrm{mmHg}$ within three minutes of standing or HUT is considered to define orthostatic hypotension. ${ }^{6}$

\section{Head-up Tilt Test}

This test complements the autonomic evaluation of active standing as, conceptually, it permits the analysis of hemodynamic modifications elicited by baroreceptor reflex activation without the interference of the muscular pump of the legs. Further it should be performed in patients with strongly suspected orthostatic dysregulation who cannot stand up actively. ${ }^{6}$ In normal subjects, a decrease in BP related to the redistribution of blood due to gravity is observed, which, in turn, elicits the activation of baroreceptors." Feedback mechanisms activate the sympathetic nervous system while decreasing parasympathetic activity in order to re-establish BP levels. Classically, hemodynamic changes associated with HUT have two phases: an initial cardiovascular acute response with a duration of 30 seconds, and a stabilization phase composed of two periods - an adaptation period occurring one to two minutes after orthostasis and a later response to prolonged orthostasis lasting for more than five minutes. ${ }^{11,12}$
This maneuver is performed on an electrically operated table. After a resting period of five minutes in the supine position, subjects are tilted, head up, to 60 to $70^{\circ}$ at a constant speed. The subject is kept upright for a period of five to 10 minutes and then returned to the supine position. Changes in BP and HR are evaluated against normative data. ${ }^{9}$ For patients with reflex syncope, HUT of 5-10 is usually not sufficient to provoke syncope. By contrast with autonomic challenge maneuvers, HUT can not only determine autonomic under-reactivity—as seen in, for example, orthostatic hypotension - it also can provoke reflex syncope. For the latter indication, the subject is tilted for up to 40 minutes. In elderly patients with a clinical history of carotid sinus hypersensitivity, HUT may be combined with massage.

\section{Analysis of Cardiovascular Signal Variability}

Variability in $\mathrm{HR}$ and $\mathrm{BP}$ also can be assessed in the frequency domain using fast fourier transform (FFT), wavelet transform (WT) or Hilbert-Huang transform $(\mathrm{HHT})$ analyses to evaluate the dynamics of sympathetic and parasympathetic innervation of the cardiovascular system. Patients with autonomic failure show different pathologic response profiles, such as delays in these adaptive responses, dysynergy between BP and HR responses and/or exaggerated responses such as orthostatic hypotension, postural orthostatic tachycardia or syncope.

Cardiovascular variables show rhythmic oscillations and stochastic fluctuations with time as a result of the summation of the cardiovascular regulatory processes. Biologic signal analysis techniques have been applied to these signals to detect pathologic patterns that can help to improve diagnoses and follow-up in autonomic disorders. In particular, the application of FFT and autoregressive spectral analysis to HR and BP signals has made a very important contribution to autonomic evaluation. FFT, by using sinus functions of different frequencies and amplitudes, decomposes the signals allowing a definition of a power spectrum where two major ranges of frequencies for human subjects can be recognized: i.e. low frequencies $(\mathrm{LF} ; 0.04-0.15 \mathrm{~Hz})$ and high frequencies (HF; 0.15-0.4 Hz). ${ }^{13}$

For oscillations in HR or RR intervals, LF has been related mainly to sympathetic outflow (although there may be also some parasympathetic influence due to slow breaths). HF is related to parasympathetic outflow and respiratory rhythm. The LF/HF ratio has been widely used as an indicator of the autonomic balance. ${ }^{14}$ However, FFT analysis shows important limitations as it requires a stationary signal and a long period of data collection. It is not useful to locate and follow changes of a frequency over time. Wavelet analysis has been used to overcome these limitations and perform a time-frequency analysis that permits correlation of LF and HF changes with any particular external signal or event. ${ }^{11,15}$

Recently, a modified HHT has been proposed as a more suitable tool for clinical autonomic evaluation, as it renders a higher time frequency resolution and better represents non-linear processes. ${ }^{16}$ These new methods have yet to be evaluated for their validity and usefulness for ANS testing.

\section{Baroreflex Sensitivity}

Evaluation of baroreceptor function (mostly assessed as baroflex sensitivity) is an important tool in the evaluation of autonomic cardiovascular control. ${ }^{12}$ 
The arterial baroreceptor reflex system regulates short-term fluctuations of arterial BP and HR. Increased systemic arterial BP activates the baroreceptors (located mainly in the carotid sinus and aortic arch walls) leading to increased cardioinhibitory vagal discharge and decreased sympathetic discharge to the heart and peripheral blood vessels and resulting in decreased $\mathrm{HR}$, cardiac contractility, peripheral vascular resistance and venous return. Decreased BP causes the opposite efferent autonomic and cardiovascular responses.

Techniques to quantify BRS include pharmacologic methods using vasoactive drugs, the Valsalva maneuver, the neck chamber technique and analysis of spontaneous fluctuations of BP and HR.

\section{Oxford Method}

This pharmacologic approach was introduced by Smyth, Sleight and Pickering. ${ }^{17}$ In this method, the alpha 1-adrenorecepor agonist, phenylephrine, is administered to induce a rapid increase in BP of between $15 \mathrm{mmHg}$ and $40 \mathrm{mmHg}$ and subsequent changes in HR are recorded. Administration of vasoconstrictor drugs primarily affects the cardiac vagal component of baroreceptor control. Modifications of the Oxford method calculate the baroreflex sensitivity after sequential injections of depressor and pressor drugs and can also help to investigate the responses of the efferent sympathetic branch. This is an invasive procedure which is contraindicated in certain patients (e.g. those with arterial hypertension). In addition, there are controversies over the selectivity associated with using vasoactive drugs to test BRS. BP stimuli might induce other reflex arcs, mainly the cardiopulmonary receptors, which interfere with BRS.

\section{Neck Chamber Technique}

The need for elaborate equipment means that this technique is mainly used in research laboratories. ${ }^{12}$ Applying negative or positive pressures to the neck allows for selective activation of carotid baroreceptors. A pressure increase within the neck chamber is sensed by the baroreceptors as a decrease in BP and vice versa. BP and HR responses are recorded.

The baroreflex is continuously active in order to keep BP at a stable level. computer-based techniques allow assessment of BRS by correlation of spontaneous fluctuations of BP with consecutive HR changes. The methods may be divided into 'time domain' (i.e. sequence) or 'frequency domain' (i.e. spectral) techniques. Sequence and spectral techniques have proven reliability and have become a standard tool in many autonomic testing devices. Clinical and scientific interpretation of results should be based on a profound knowledge on the technique applied, however, and special care should be taken not to interpret measurements influenced by artifacts or ectopic activity.

\section{Sequence Techniques}

A number of different sequence techniques have been published. Sequence techniques evaluate BRS by correlation of BP increase with subsequent HR decrease or vice versa. Significant agreement has been demonstrated between the sequence method and the 'gold standard' phenylephrine method. The methods are easy to apply and do not require the co-operation of the patient. Since there is no load on BP, only a small portion of the baroreceptor reflex is evaluated. Various sequence methods yield different results when applied to the same data sets, particularly in patients with impaired baroreflex function. ${ }^{18,19}$ Therefore, the method should be selected carefully with regard to the pathophysiologic background of the patient tested.

\section{Spectral Techniques}

Evaluation of BRS by spectral techniques is based on the concept that spontaneous oscillations in BP result in spontaneous oscillations in HR within the same frequency range. Two frequency bands are of interest: the low frequency band around $0.1 \mathrm{~Hz}$ and the high frequency band around the respiratory frequency. Several algorithms have been developed to calculate spectral indices. Newer methods have demonstrated the capability of detecting impairment of BRS in structural cardiovascular disease. There is controversy over the comparability of these new techniques with the phenylephrine test. This might be explained by methodologic differences: spectral algorithms focus on oscillatory changes around a set point, whereas the phenylephrine test relies on a strong unidirectional response. ${ }^{12}$ Interference from artifacts (noise, ectopic activity, etc) is a major limitation to any spectral technique. In patients with depressed BP variability, the signal-to-noise ratio tends to become high and influence BRS reliability. Further limitations have been described previously.

\section{Sudomotor Function}

Disorders of sweating (hypo- or hyperhidrosis) can be focal or generalized and appear quite frequently in ANS failure. Evaluation of sudomotor function can provide early diagnosis of small fiber neuropathy, ${ }^{20}$ particularly in early diabetes, and is used to provide a measure of cholinergic sympathetic function. A large number of different tests are available. They can assess central and peripheral sudomotor function, as the thermoregulatory sweat test (TST) ${ }^{21}$, or postganglionic function alone, as the quantitative sudomotor axon reflex test (QSART), ${ }^{22}$ the sympathetic skin response test (SSRT), the quantitative direct and indirect axon reflex test (QDIRT) ${ }^{23}$ and the dynamic sweat test (DST). ${ }^{24}$ Patterns of sweating can be visualized by topical application of indicators such as iodinate starch or sodium alizarin sulfonate after a sufficient thermal stimulus has been applied. Quantitative tests as QSART, QDIRT, DST, SSRT and the silastic imprint test (SIT) $)^{25}$ may be used to study sweat gland activity in more detail.

\section{Thermoregulatory Sweat Test}

This test can assess central and peripheral sudomotor function ${ }^{21}$ and is performed in a temperature $\left(45-50{ }^{\circ} \mathrm{C}\right)$ and humidity controlled chamber. The whole body is covered with an indicator dye and the change in color due to sweat production is documented. Asymmetric patterns due to focal anhidrosis or stocking- and glove-like distributions may be observed in length-dependent neuropathy. Limitations result from the visual analysis and the lack of differentiation between pre- and postganglionic lesions. The test is time-consuming and needs special equipment.

\section{Quantitative Sudomotor Axon Reflex Test}

This test measures post-ganglionic axon reflex-mediated sweat production in a small restricted area of the skin over time.22 A multicompartmental sweat capsule is used to stimulate sweat glands by iontophoresis of acetylcholine. A first, direct sweat response in the area of iontophoresis is discriminated from the indirect, axon reflex-mediated response in the surrounding area (for details, see the review by Illigens and Gibbons ${ }^{20}$ ). 
Sweat can be sampled over a time period and the results of the analysis correlated with other stimuli. Limitations are the expensive equipment, the very small detection area and the inability to detect preganglionic lesions.

\section{Silastic Imprint Test}

The test evaluates postganglionic sympathetic cholinergic sudomotor function by measuring the direct and axon-reflex mediated sweat response. ${ }^{25}$ Sweat glands are stimulated by iontophoresis of acetylcholine, pilocarpine or methacholine. This is followed by application of a thin layer of moldable silastic material to the skin. Sweat droplets displace the silicone material during polymerization, resulting in permanent impressions in the silastic material that can be quantified using various methods. Number of droplets, size and distribution are reported.

Although this is probably the easiest method of quantifying sudomotor function, limitations include the dependence of the results on the material used (polymerization time, added surfactants, etc.) and its susceptibility to artefacts such as hairs, dirt, air bubbles, and skin surface texture. This method has a good spatial resolution, but renders no temporal information.

\section{Quantitative Direct and Indirect Axon Reflex and Direct Sweat Tests}

The QDIRT ${ }^{23}$ and DST ${ }^{24}$ quantify sudomotor function with spatial and temporal resolution. They each combine stimulation of sweat glands and sudomotor axons by iontophoresis of a cholinergic agonist into the skin with use of a colored indicator of sweat release. Each sweat droplet results in a color spot. The spots increase in size and number over time and the evolving pattern of spots is recorded by high resolution digital photography or video and is evaluated using automated image analysis software.

Limitations are the sensitivity of the results to ambient temperature and humidity and the patient's hydration status and caffeine intake. The problem of sweat evaporation might be resolved by DST by adopting a cornstarch-powdered waterproof transparent tape. Both methods only recently have been described and need further investigation to prove their reproducibility and sensitivity and to establish normative data.

\section{Sympathetic Skin Response Test}

This measure of electrodermal activity provides a surrogate marker of sympathetic cholinergic sudomotor activity. An arousal stimulus (electric, acoustic, deep breath) induces a change in skin potential, which usually is recorded from the palms and soles of the feet. SSRs are reported as present or absent. Normative values for amplitude and latency have been published. Although this test is very easy to perform and has been integrated in many commercial EMG devices, there are serious limitations. These include habituation, high intra- and inter-individual variability, and the fact that SSR declines with age.

\section{Summary and Future Perspectives}

Autonomic nervous system disorders are associated with a severe disease burden, it is therefore of utmost importance that they are diagnosed. In many cases the diagnosis can be established by history taking and simple bedside tests. In a number of cases, however, precise cardiovascular and sudomotor tests are necessary to confirm the diagnosis. This review describes widely used techniques.

Currently, no gold standards for autonomic testing are available. Differences in tests performed are an obstacle for comparing scientific and clinical data acquired in different labs. We hope that this review proves to be a valuable help in focusing on standard tests in autonomic diagnosis.

Growing recognition of ANS disorders increases also the number of neurologic colleagues involved in the field. Every neurologist should be able to diagnose autonomic diseases based on the history. Although a full featured laboratory is difficult to finance, we envision that autonomic testing becomes available at least in central hospitals in many countries. Standards for autonomic testing have to be developed in the nearest future.
1. Lahrmann $\mathrm{H}$, Magnifico F, Haensch CA, Cortelli P, Autonomic nervous system laboratories: a European survey, Eur I Neurol, 2005;12:375-9.

2. Moya A, Sutton $R$, Ammirati $F$, et al., Guidelines for the diagnosis and management of syncope (version 2009): The Task Force for the Diagnosis and Management of Syncope of the European Society of Cardiology (ESC), Eur Heart J, 2009;30:2631-71.

3. Thijs RD, van Dijk JG, Bloem BR, Falls, fits, faints and funny turns, I Neurol, 2009;256:155-67.

4. van Dijk N, Boer KR, Colman N, et al., High diagnostic yield and accuracy of history, physical examination, and ECG in patients with transient loss of consciousness in FAST: the fainting assessment study, I Cardiovasc Electrophysiol, 2008:19:48-55.

5. Freeman R, Clinical practice. Neurogenic orthostatic hypotension, N Eng/ J Med, 2008;358:615-24.

6. Lahrmann, $\mathrm{H}$, Cortelli $\mathrm{P}$, Hilz M, et al., EFNS guidelines on the diagnosis and management of orthostatic hypotension, Eur J Neurol, 2006;13:930-6.

7. Winker R, Prager W, Haider A, et al., Schellong test in orthostatic dysregulation: a comparison with tilt-table testing, Wien Klin Wochenschr, 2005;117:36-41.

8. Ewing DJ, Clarke BF, Diagnosis and management of diabetic autonomic neuropathy, Br Med J, 1982;285:916-8.

9. Low PA, Sletten DM, Laboratory evaluation of autonomic failure. In: Low PA, Benarroch EE (eds), Clinical Autonomic Disorders: Evaluation and Management, Philadelphia: Lippincott Williams \& Wilkins, 2008;130-63.
10. The Consensus Committee of the American Autonomic Society and the American Academy of Neurology, Consensus statement on the definition of orthostatic hypotension, pure autonomic failure and multiple system atrophy, Neurology, 1996:46:1470

11. Ducla-Soares JL, Santos-Bento M, Laranjo S, et al., Wavelet analysis of autonomic outflow of normal subjects on head-up tilt, cold pressor test, Valsalva manoeuvre and deep breathing, Exp Physiol, 2007;92:677-86.

12. Hilz MJ, Dutsch M, Quantitative studies of autonomic function, Muscle Nerve, 2006;33:6-20.

13. Task Force of the European Society of Cardiology, and the North American Society of Pacing and Electrophysiology, Heart rate variability, standards of measurement, physiological interpretation and clinical use, Circulation, 1993,93:1043-65.

14. Malliani A, Pagani M, Lombardi F, Cerutti S, Cardiovascular neura regulation explored in the frequency domain, Circulation, 1991:84:482-92.

15. Postolache G, Rocha I, Postolache O, et al., A wavelet based approach for monitoring baroreceptors function test in rats, In: Proceedings of the Instrumentation and Measurement Technology Conference, LOS Alamitos, CA: IEEE 2004:844-9.

16. Tavares $\mathrm{C}$, Laranjo $\mathrm{S}$, Santos $\mathrm{M}$, et al., An instantaneous time-frequency methodology applied to evaluation of blood pressure changes during HUT of multiple system atrophy patients, CAR, 2010;20:150-1.

17. La Rovere MT, Pinna GD, Raczak G, Baroreflex sensitivity: measurement and clinical implications, Ann Noninvasive Electrocardiol, 2008:13:191-207.

18. Laude D, Elghozi JL, Girard A, et al., Comparison of various techniques used to estimate spontaneous baroreflex sensitivity (the EuroBaVar study), Am J Physiol Regul Integr Comp Physiol, 2004;286:226-31

19. Struhal W, Hoedl $S$, Lahmann $H$, et al., Reliability of the noninvasive sequence methods to estimate baroreflex function using the paradigm of brain death, Auton Neurosci, 2010; [Epub ahead of print].

20. Illigens $\mathrm{BM}$, Gibbons $\mathrm{CH}$, Sweat testing to evaluate autonomic function, Clin Auton Res, 2009:19:79-87.

21. Fealey RD, LoW PA, Thomas JE, Thermoregulatory sweating abnormalities in diabetes mellitus, Mayo Clin Proc, 1989;64:617-28.

22. Low PA, Caskey PE, Tuck RR, et al., Quantitative sudomotor axon reflex test in normal and neuropathic subjects, Ann Neurol, 1983:14:573-80

23. Gibbons $\mathrm{CH}$, Illigens BMW, Centi J, Freeman R, QDIRT: quantitative direct and indirect test of sudomotor function, Neurology, 2008;70;2299-304.

24. Provitera V, Nolano M, Caporaso G, et al., Evaluation of sudomotor function in diabetes using the dynamic sweat test, Neurology, 2010;74:50-6.

25. Stewart JD, Nguyen DM, Abrahamowicz M, Quantitative sweat testing using acetylcholine for direct and axon reflex mediated stimulation with silicone mold recording; controls versus neuropathic diabetics, Muscle Nerve, 1994;17:1370-7. 